\title{
Electronic band structure of calcium oxide
}

\author{
M A Bolorizadeh ${ }^{* \S}$, V A Sashin $\dagger$, A S Kheifetsł and M J Ford $\dagger^{*}$ \\ †School of Chemistry, Physics and Earth Science, Flinders University of South Australia, \\ GPO Box 2100, Adelaide, SA 5001, Australia \\ ${ }^{\S}$ Physics Department, Shahid Bahonar University, Kerman, Iran. \\ $\ddagger$ Research School of Physical Sciences and Engineering, Institute of Advanced Studies, ANU, \\ Canberra ACT 0200, Australia. \\ \#Institute for Nanoscale Technology, University of Technology, Sydney, PO Box 123, \\ Broadway, NSW 2007, Australia
}

\begin{abstract}
We employed electron momentum spectroscopy (EMS) to measure the bulk electronic structure of calcium oxide. We extracted the electron momentum density (EMD), density of occupied states (DOS), band dispersions, bandwidths and intervalence bandgaps from the data. The results are compared with calculations based on the full potential linear muffin-tin orbital (FP-LMTO) approximation. While the bandwidths of $0.6 \pm 0.2 \mathrm{eV}$ and $1.2 \pm$ $0.1 \mathrm{eV}$ for the $s$ - and $p$ - bands, respectively, and their dispersions agree well with the LMTO calculation, the relative intensity of the two bands is at odds with the theory. The measured intervalence bandgap at the $\Gamma$-point of $16.5 \pm 0.2 \mathrm{eV}$ is larger by $2.1 \mathrm{eV}$ than that from the LMTO calculation. The experimental bandwidth of the Ca $3 p$ semi-core level of $0.7 \pm 0.1 \mathrm{eV}$ agrees with the LMTO prediction. The measured bandgap between this level and the $s$-band is $3.6 \pm 0.2 \mathrm{eV}$. The Ca $3 s-3 p$ level splitting is in excellent agreement with the literature.
\end{abstract}

Keywords: Alkaline earth methals; electron momentum spectroscopy; Electron-solid scattering and transition-inelastic

\footnotetext{
*Corresponding author: mabolori@mail.uk.ac.ir
} 


\section{Introduction}

The light alkaline-earth oxides (AEOs) form a chemically interesting series of metal oxides. Calcium oxide is the heaviest one in this group and considered to be a purely "ionic" solid. Knowledge of the electronic properties of the AEOs is essential due to their rapidly increasing technological importance for production of new materials. Characteristics of the AEOs such as high mechanical and radiation resistance strengths, transparency in the infrared, visible and vacuum ultraviolet regions of the spectrum, open new prospects for their application in high temperature techniques, microelectronics and heterogeneous catalysis, to name a few. They are also of geophysical interest [1], since $\mathrm{MgO}$ and $\mathrm{CaO}$ are present in significant amounts in the lower mantle of the Earth. At normal conditions $\mathrm{CaO}$ crystallizes into the cubic rocksalt $(\mathrm{NaCl})$ structure and is considered as a prototype oxide from the theoretical point of view. This compound exhibits properties typical of an insulator, with a wide bandgap of $7.7 \mathrm{eV}$ [2] and also of a semiconductor, with a high dielectric constant of 11.8 .

In a simple ionic model, solid state $\mathrm{CaO}$ is formed by an array of cations, $\mathrm{Ca}^{2+}$, and anions, $\mathrm{O}^{2-}$, with highly ionic bonding between them. Despite the obvious success of this model [3], it fails to correctly predict the bandgap of this ionic solid, partly due to the presence of some degree of covalency in the bonding. A competing picture is based on a model postulating delocalization of one electron transferred from the cation to the anion $\left(\mathrm{Ca}^{2+} \mathrm{O}^{-}\right.$plus $\left.\mathrm{e}^{-}\right)[4,5]$. The alternative model is supported by two factors: (a) the low-lying empty $3 d$ orbitals of the cation [6] and (b) the instability of the $\mathrm{O}^{2-}$ ion due to an insufficiently strong Madelung potential [7].

A number of band structure calculations on $\mathrm{CaO}$ have been carried out by various theoretical approaches $[8,9,10,11,12,13,14,15,16,17,18,19,20]$. At the same time experimental data on the subject are quite limited. O'Bryan and Skinner [21] measured the 
K-emission spectra of Ca using soft X-rays and obtained the first estimate of bandwidth of 15 $\mathrm{eV}$ for the upper valence sub-band. Several authors $[22,23,24]$ made XPS measurements of the valence band and $\mathrm{Ca} 3 p$ and $3 s$ semi-core levels. We note that a few optical measurements were also performed on $\mathrm{CaO}$ to probe the conduction and valence band structures (see, for example, Whited and Walker [25]). There is also a band structure measurement by the UV diffuse reflectance method [26]. However, none of the abovementioned experimental techniques delivers the full energy-momentum resolved band structure, which is quite important for an accurate description of the electronic properties. Angle resolved photoemission spectroscopy (ARPES) could provide the full band structure information, but to the best of our knowledge there are no reported ARPES measurements of $\mathrm{CaO}$.

We used electron momentum spectroscopy (EMS) [27] to study the valence and corelevel structure of $\mathrm{CaO}$ films. EMS can provide a direct measurement of electron distribution in energy-momentum space, and has been applied to a variety of targets in gaseous [28,29] and solid $[30,31,32,33]$ forms. EMS allows us to directly observe the dispersion relationship in solids, and also measure band intensities, i.e. the full band structure of a solid target.

In this paper we report on the valence band and $\mathrm{Ca}$ semi-core levels measured in $\mathrm{CaO}$. The experimental results are compared to theoretical calculations using the full potential linear muffin-tin orbital (FP-LMTO) approximation and also to the results of other researchers. This comparison is thought to serve as a sound basis for further improvement in the theoretical description of ionic solids.

In the next two sections we briefly describe our $(e, 2 e)$ spectrometer, followed by the target preparation and characterization techniques used. A short outline of the FT-LMTO method and theoretical results for crystalline $\mathrm{CaO}$ is given in section 3. Section 4 deals with 
the results of the experiment and their comparison to theory. Concluding remarks are presented in section 5 .

\section{Experimental Technique}

\section{1. (e,2e) Spectrometer}

Details of the $(\mathrm{e}, 2 \mathrm{e})$ spectrometer have been published previously in the literature [34]. However, we will present a short description of the spectrometer and the main principles behind EMS. Consider an energetic incident electron with energy $E_{0}$ and momentum $\mathbf{p}_{0}$ striking a target and knocking out an electron. At sufficiently high incident energy and momentum transfer the collision can be regarded as binary and the plane-wave impulse approximation (PWIA) can be used. The two outgoing electrons are detected in coincidence to ensure that they originated from the same $(e, 2 e)$ event. Measuring their energies and momenta the binding energy $\varepsilon$ and momentum $\mathbf{q}$ of the target electron before the collision can be determined from the following conservation laws:

$\varepsilon=E_{0}-E_{\mathrm{s}}-E_{\mathrm{f}}$

$\mathbf{q}=\mathbf{p}_{\mathrm{s}}+\mathbf{p}_{\mathrm{f}}-\mathbf{p}_{0}$,

where $\left(E_{\mathrm{f}}\right.$ and $\left.\mathbf{p}_{\mathrm{f}}\right)$ are the energy and momentum of the fast electron, and $\left(E_{\mathrm{s}}\right.$ and $\left.\mathbf{p}_{\mathrm{s}}\right)$ are the energy and momentum of the slow electron; i.e. the $(e, 2 e)$ technique is kinematically complete. In the PWIA, the (e,2e) cross section is proportional to the modulus squared of the target electron momentum space wavefunction $\psi(\varepsilon, \boldsymbol{q})[28,35]$, in other words the electron spectral momentum density (SMD). Thus measurement of the (e,2e) cross section at different $\varepsilon$ and $\boldsymbol{q}$ is a direct mapping of the SMD of the bound electrons in the target. 
Fig. 1(a) shows the scattering geometry of our (e,2e) spectrometer which is characterized by non-coplanar asymmetric kinematics to ensure a high coincidence count rate and good energy and momentum resolution. The incident and two outgoing electron energies are nominally $20.8 \mathrm{keV}, 19.6 \mathrm{keV}$ and $1.2 \mathrm{keV}$, respectively. The outgoing electrons are detected by electrostatic analysers located at polar angles of $13.6^{\circ}$ and $76^{\circ}$ relative to the direction of the incident beam on the opposite side of the target from the incident beam. The overall momentum detection range is about \pm 3.5 a.u.. The overall resolution is about $1 \mathrm{eV}$ in energy and 0.15 a.u. in momentum of the target electron. The spectrometer maps the SMD of target electrons whose momenta are directed along the $y$-axis.

An important characteristic of our spectrometer is that it is most sensitive to the outermost $2 \mathrm{~nm}$ surface layer of the target, as shown in Fig. 1(b) by shading. This results from a small escape depth of the slow electron. Hence most electronic structure information comes predominantly from this area. This feature facilitates measurement of targets prepared by evaporation onto a thin substrate, (for instance, an amorphous carbon film), with minor contribution to the signal. The contribution from carbon substrate could easily be subtracted from the spectrum, while (e,2e) events occurring deeper within the target (non-shaded area) contribute mainly to the background intensity. The thickness of solid targets must not exceed about $10 \mathrm{~nm}$, otherwise, background events due to multiple scattering in the target dominate the $(e, 2 e)$ signal [36].

\subsection{Sample Preparation and Characterization}

We followed the target preparation technique used for $\mathrm{MgO}$ [31]. Calcium (99\% pure) was resistively heated and deposited onto annealed amorphous carbon (am-C) substrates $(3 \mathrm{~nm}$ thick) in a $10^{-6}$ Torr oxygen background. A slow evaporation rate (about $0.2 \mathrm{~nm} / \mathrm{min}$.) allowed sufficient time for oxidation of the deposited Ca layer. The optimum thickness of the 
formed layer is determined on the basis of providing a complete coverage of the am-C substrate surface and not so thick that multiple scattering dominates. It has been found that a $3 \mathrm{~nm}$ thick $\mathrm{CaO}$ layer satisfies both conditions. The structural character of the formed $\mathrm{CaO}$ films is believed to be polycrystalline[37].

Typical Auger spectra recorded for a pure $\mathrm{Ca}$ and an as-prepared $\mathrm{CaO}$ surface are presented in Figs. 2 (a) and (b), respectively. A clear transformation in the pure Ca Auger spectrum indicates oxide formation. Firstly, a prominent $\mathrm{O}$ KVV line at about $525 \mathrm{eV}$ has appeared (note that the energy scale is shifted about $10 \mathrm{eV}$ due to a charge build up on the target by a relatively high Auger electron gun current [38] compared to the one used in our (e,2e) experiment). The pioneering work of Ascarelli, et al [39] and the recent studies of Cazaux [40] show that charge build up should be considered for thick insulating target having an escape depth of about $100 \mathrm{~nm}$ in electron spectroscopy. Due to small thickness of our sample and low current of the primary beam, we expect that charging effect is negligible in the $(\mathrm{e}, 2 \mathrm{e})$ experiment as opposed to the Auger spectrum. Secondly, as a result of electron transfer from the calcium cations to the oxygen anions, the Auger transitions which involve the valence electrons (i.e., the $\mathrm{Ca}_{2,3} \mathrm{VV}$ and $\mathrm{L}_{2,3} \mathrm{M}_{2,3} \mathrm{~V}$ lines) have been strongly attenuated. This is particularly clear for the $\mathrm{Ca} \mathrm{M}_{2,3} \mathrm{VV}$ line which is shown in detail in the inset. In addition, the shape of the four $\mathrm{L}_{2,3} \mathrm{M}_{2,3} \mathrm{M}_{2,3}$ lines, in the energy range from $292 \mathrm{eV}$ to $303 \mathrm{eV}$, have also changed dramatically due to the different chemical environment of Ca.

We can see no clear indication of the presence of carbon on the sample surface as shown in Fig. 2(b). However, the structure about $280 \mathrm{eV}$ may hint at the presence of carbon (the literature value is $275 \mathrm{eV}$ for the $\mathrm{C} \mathrm{KVV}$ line [41]). We will see shortly that the EMS data show only a negligible input from the am-C substrate. The origin of small broad structures (marked by arrows) observed about $35 \mathrm{eV}$ down in energy from the intense $\mathrm{Ca}$ $\mathrm{L}_{2,3} \mathrm{M}_{2,3} \mathrm{M}_{2,3}$ and $\mathrm{O}$ KLL lines is thought to be due to a $35 \mathrm{eV}$ energy loss of Auger electrons 
in $\mathrm{CaO}$. The band structure calculations assign this loss to an interband transition between the valence and conduction bands (see, for example Mattheiss [42]). The same energy loss feature is also visible in the pure Ca spectrum.

We also performed the electron energy loss (EEL) measurement of the as-prepared $\mathrm{CaO}$ target to understand the effect of multiple scattering processes in the EMS data. The result is shown in Figs. 3 (a) and (b) for $19.6 \mathrm{keV}$ and $1.2 \mathrm{keV}$ electrons, respectively. Both spectra are normalized at the elastic peak. Note that the $19.6 \mathrm{keV}$ electron spectrum comprises data from several $\mathrm{CaO}$ targets of different thickness (from 3 to $10 \mathrm{~nm}$ ). One can distinguish three energy loss structures in this spectrum. A broad peak centred at about 22.5 $\mathrm{eV}$ is due to am-C bulk plasmon losses. There are two more peaks in the spectrum, at around $14 \mathrm{eV}$ and $37 \mathrm{eV}$, which probably originate from losses due to excitation of valence electrons to low lying conduction bands in $\mathrm{CaO}$. However, the free-electron model plasmon energy, $\hbar \omega_{p l \text {, }}$ associated with the six electrons of the upper valence sub-band, is about $17 \mathrm{eV}$. As this is only $3 \mathrm{eV}$ off the energy of the first $\mathrm{CaO}$ peak (at $14 \mathrm{eV}$ ), this feature may be of plasmon origin. The $1.2 \mathrm{keV}$ electron spectrum in Fig. 3(b), from a $3 \mathrm{~nm}$ thick as-prepared $\mathrm{CaO}$ target, also shows the same peaks. In this case, however, they are not as easily observed as in Fig. 3(a).

We obtained the precise positions and widths of the energy loss features by applying an appropriate least squares fit to the $19.6 \mathrm{keV}$ electron spectrum. The fitting function was a linear combination of three Gaussians and a second order polynomial to approximate the smooth background. The position of the am-C plasmon peak was fixed at $22.5 \mathrm{eV}$. The result of this process is represented by a solid line in Fig. 3(a) and the parameters being 13.7 $\pm 0.1 \mathrm{eV}$ and $1.7 \pm 0.3 \mathrm{eV}$ for the position and half-width (FWHM), respectively, of the first energy loss peak. For the second peak the corresponding values are $36.6 \pm 0.2 \mathrm{eV}$ and $4.5 \pm$ $0.5 \mathrm{eV}$. Similar energy loss peaks were observed by Rudberg $[43,44]$ for the inelastic 
scattering of electrons in 'aged' Ca films on silver and platinum substrates. The average peak positions of the two Ca deposits, extracted from Fig. 10 of the paper by Rudberg [43], are $13.6 \mathrm{eV}$ and $36.5 \mathrm{eV}$. Protheroe and co-workers [45] using low energy electrons (from 200 $\mathrm{eV}$ to $1300 \mathrm{eV}$ ) found a number of peaks in the CaO EEL spectrum, positions of two of them at energies of $13.1 \mathrm{eV}$ and $35.0 \mathrm{eV}$ are close to the present result. The work of Whited and Walker [25] who obtained the energy-loss function, $\operatorname{Im} \varepsilon^{-1}$, for $\mathrm{CaO}$ from the reflectance spectra also supports our data. This function shows a large peak at $35 \mathrm{eV}$ and three small peaks at about $14 \mathrm{eV}, 18 \mathrm{eV}$ and $27 \mathrm{eV}$.

\section{LMTO Calculation}

The band structure and electron momentum density (EMD) were calculated using the FPLMTO method, as described in Kheifets et al. [46]. The local density approximation (LDA) was employed with the Janak-Moruzzi-Williams parameterization to the exchangecorrelation functional. The experimental lattice parameter $a=4.8105 \AA$ was used, according to Wyckoff [47]. The fcc unit cell of $\mathrm{CaO}$ has two non-equivalent atomic positions at $(0,0,0)$ and $(1 / 2,1 / 2,1 / 2)$, occupied by the $\mathrm{Ca}$ and $\mathrm{O}$ atoms, respectively. The two non-overlapping muffin-tin spheres of radius $a / 4$ were placed at these positions.

The calculated energy bands (two top rows) and EMDs (bottom row) of $\mathrm{CaO}$ in the [100], [110] and [111] high symmetry directions, together with the first Brillouin zone, are shown in Fig. 4. The EMDs are plotted with the same line styles as used for the energy bands. The lower and upper valence sub-bands are formed by hybridization of the $\mathrm{O} 2 s^{2}$ valence electrons with the $\mathrm{Ca} 3 p^{6}$ semi-core electrons and the $\mathrm{O} 2 p^{4}$ with the $\mathrm{Ca} 4 s^{2}$ valence states, respectively. The dotted lines show the three lowest bands derived from the $\mathrm{Ca} 3 p^{6}$ semicore states, the thick dashed line indicates the lower $\mathrm{O} 2 s$ band and the thin solid lines 
show the three upper $p$-bands. The momentum densities of the $p$-like bands are zero at the $\Gamma$ point. The momentum density of the $s$-like bands is at its maximum at zero momentum thereafter it falls quickly with increasing momentum leading to a secondary maximum. This secondary maximum indicates a contribution of the Ca $3 p$ orbitals to this band. The local minimum of the $s$-momentum density almost coincides with the maximum of the $p$ momentum density.

We have measured the band dispersions, bandwidths and bandgaps using our experimental SMD and the LMTO calculations. In order to compare properly the experimental results with the LMTO calculation, two consecutive transformations of the theoretical SMD was performed to simulate the experimental conditions, which we call expcorrected calculation. First of all, the LMTO intensity was spherically averaged over the irreducible wedge of the first Brillouin zone to account for the polycrystalline nature of the measured target. Secondly, the LMTO intensity was corrected for the spectrometer resolution in the energy and momentum directions by Gaussians of half-widths of $1 \mathrm{eV}$ and 0.15 a.u., respectively. In Fig. 5(a), we present the theoretical SMD spherically averaged and corrected for the spectrometer resolution. As the energy is referenced to the vacuum level of the experiment, it is shifted in fig. (5a) to match the s-band minimum in the experiment.

The experimental results are subjected to other effects such as multiple scattering and misalignment of the spectrometer. Elastic scattering mainly causes a smearing of intensity along the momentum axis while inelastic scattering is responsible for intensity distributed along the energy axis [48]. Multiple scattering was simulated into the theory by an additional energy and momentum convolution of the LMTO data only to calculate the bandgap, bandwidth and band dispersions. The parameters of this convolution were chosen in such a way as to obtain peak widths in the calculation similar to those in the experiment at the momenta corresponding to the band extrema. This is, however, a very ad hoc method for 
incorporating the effects of multiple scattering and the agreement may be fortuitous. Our attempt to account for this by simple additional convolution of the calculation improves the agreement for measured bandwidth, while sometimes deteriorates the agreement for measured intervalence bandgap. A proper Monte Carlo (MC) simulation of multiple scattering in the target is needed to shed more light on these discrepancies However we do not recommend this in the LDA calculations. Multiple scattering effects were implemented into LCAO calculations at the GGA and hybrid level and compared with our BeO results [49] but multiple scattering does not completely explain all the discrepancies.

Misalignment of the spectrometer had a minor effect on the experimental results. The spectrometer geometry has been aligned carefully by repeating measurements on am-C films and thus we are confident that the influence of the residual misalignment on the experimental results has been minimised.

\section{Results and Discussion}

\subsection{Valence Band}

The experimental SMD of the valence band and core levels of $\mathrm{Ca}$ in $\mathrm{CaO}$ is shown in Fig. 5(b) as a grey scale plot. Energy is referenced to the vacuum level of the spectrometer. One can observe structures typical to ionic solids, at about $8 \mathrm{eV}$ and $24 \mathrm{eV}$, derived predominantly from the $2 p$ and $2 s$ levels of oxygen, respectively. The upper ( $p$-band) and lower ( $s$-band) valence sub-bands are separated by an intervalence bandgap of about $14 \mathrm{eV}$. The $p$-band consists of two symmetrical branches with their maximum density away from the $\Gamma$ point, whereas the $s$-band has its maximum at the $\Gamma$ point. The $3 p$ core level of $\mathrm{Ca}$ is located at about $28 \mathrm{eV}$, which is only about $4 \mathrm{eV}$ below the $s$-band. It has a small, but still noticeable, dispersion. For this reason, this level is sometimes called a semi-core level. At last, intensity around $47 \mathrm{eV}$ is due to the $3 \mathrm{~s}$ core level of $\mathrm{Ca}$. It exhibits no energy dispersion. 
Some additional intensity is also present outside the above-mentioned structures due to multiple scattering of $(\mathrm{e}, 2 \mathrm{e})$ electrons in the target. Fortunately, the energy loss spectrum of $\mathrm{CaO}$ (see Fig. 3) does not display any strong collective plasma oscillation. The $14 \mathrm{eV}$ and $37 \mathrm{eV}$ peaks are not intense enough to produce any peak-like features in the SMD, and so this background is mostly smooth.

A faint parabolic feature, having its minimum superimposed onto the $s$-band and spreading nearly to the $p$-band belongs to the $\sigma$-valence band of am-C [50]. A much weaker intensity from the $\pi$-bands of am-C is superimposed onto the $p$-band. However, this background interferes mainly with the $s$-band. To remove this background we scaled the previously measured am-C SMD and subtracted it from the measured intensity as shown in Fig. 5(c). Comparing Figs. 5(a) and (c) one can conclude that the experimental and theoretical band structures are qualitatively alike, although there are two substantial quantitative differences.

The first difference is related to the relative intensity of the two valence sub-bands. In the experiment, the intensity of the $p$-band is much higher than that of the $s$-band, while the situation is reverse for the LMTO calculation. The second major difference is related to the magnitude of intervalence bandgap, which is more than $2 \mathrm{eV}$ smaller in the theory than that in the experiment.

To remove the background and in this way to make a proper comparison of the experimental and theoretical data, we performed least squares fits to the intensity of binding energy profiles obtained by integration of the SMD intensity in Fig. 5(c) over 0.05 a.u. momentum wide slices. The positive and negative momentum components of the SMD were summed to improve the statistics, as it is expected to be symmetric about the $\Gamma$ point. The fitting function was a combination of four Gaussians for the peaks and a straight line for the smooth background. 
The background subtracted experimental as discussed in the previous paragrph and LMTO binding energy profiles for the valence band, at 0.2 a.u. intervals, are compared in Fig. 6. The momentum width of each profile is 0.05 a.u.. Intensities are normalized for the $s$-band at 0 a.u.. Comparing widths of the $s$-band peaks from the experiment and theory one can see that the experimental widths are at least two times broader than those of the LMTO calculation. Multiple scattering in the experiment is considered, at least partially, responsible for the observed additional broadening. Another source of this broadening is expected from electron correlation effects due to finite lifetimes. Comparing peak heights, the LMTO peak height drops faster than that of the experiment for small momenta, up to about 0.6 a.u.. For higher momenta the experiment-to-theory height ratio is, however, close to a constant of 2 .

As noted earlier, the intervalence bandgap in the experiment is larger, by as much as about $2 \mathrm{eV}$, than that of the theory. Hence there is a shift of about $2 \mathrm{eV}$ in positions of the corresponding $p$-band peaks. A similar increase of the experimental bandgap was observed in our previous measurements of $\mathrm{MgO}$ [31] and $\mathrm{BeO}$ [51]. This discrepancy seems to be due to the failure of the LDA approximation to correctly describe electron correlation effects in insulating solids. More sophisticated schemes, like GCA and hybrid approximations, give a better agreement with the experiment [49]. The LMTO calculation predicts no intensity at the $\Gamma$ point, typical for $p$-like orbitals, but the experiment shows an intense broad structure of a width of approximately $2.5 \mathrm{eV}$ with a height even larger than that of the $s$-band peak at 0 a.u.. It is difficult to see how elastic scattering could be entirely responsible for this intensity at the $\Gamma$ point. One of the factors contributing to this intensity may be due to a small misalignment of the spectrometer scattering geometry. Finally we note one other discrepancy between the measurement and LMTO calculation for the $p$-band. That is, while the experimental $p$-band has discernible intensity up to a momentum of 2.6 a.u., the LMTO result appears to give noticeable intensity only up to 2 a.u.. 


\subsection{1. $\underline{\text { s-Band }}$}

In order to extract the $s$-band dispersion, we performed least squares fits to the peak tops in binding energy profiles similar to those in Fig. 6. A Gaussian with three variable parameters was used. The position of the fitted peak maximum as a function of momentum, i.e. the dispersion is shown in Fig. 7(a). One can see that the experimental data disperses approximately from $24.1 \mathrm{eV}$, at the $\Gamma$ point, to $23.5 \mathrm{eV}$ at 0.55 a.u.. For a momentum range from 0.6 to 0.9 a.u. the dispersion points go slightly backwards to about $23.9 \mathrm{eV}$ and thereafter they are approximately constant up to 1.5 a.u.. The LMTO curve disperses from about 24.1 to $23.3 \mathrm{eV}$ over the momentum range from 0 to 0.5 a.u.. It is basically flat at higher momenta ( $>0.8$ a.u.). One would conclude that the experimental and exp-corrected LMTO dispersions do not agree well.

The bandwidth (defined as the difference between the minimum and maximum energies of the dispersion curve) is $0.6 \pm 0.2 \mathrm{eV}$ and $0.82 \mathrm{eV}$ for the experiment and expcorrected LMTO calculation, respectively. The full bandwidth predicted by the LMTO calculation (as extracted from Fig. 4) is $1.35 \mathrm{eV}$. The parameters of the convolution to account for multiple scattering were chosen in such a way as to obtain peak widths in the calculation similar to those in the experiment at the momenta corresponding to the band extrema, i.e. at the $\Gamma$ point and 0.55 a.u.. After accounting for multiple scattering, the LMTO bandwidth becomes $0.66 \mathrm{eV}$, which is in good agreement with the experimental value.

The experimental and theoretical EMDs for the $s$-band are shown in Fig. 7(b). Both sets of data are normalized to unity and show their maxima around the $\Gamma$ point, which is typical for $s$-like orbitals. The experimental data exhibits higher values throughout most of the momentum range, especially with a larger difference for momenta over 0.6 a.u.. As noted earlier, for this momentum range the experimental EMD is strongly enhanced by elastic 
scattering and, say at a momentum of 1 a.u. exceeds the LMTO by a factor of 2 . Besides, the presence of a strong Ca $3 p$ signal in this energy region makes it difficult to correctly evaluate the s-band intensity. However, a pattern of the theoretical EMD (a characteristic flattening in the 0.5 - 1 a.u. momentum interval due to strong hybridization of this band with the Ca $3 p$ orbitals) is still discernible in the experimental result.

We compare our results with the bandwidths calculated by Stepanova and her coworkers [9] using the linear augmented plane waves (LAPW) approximation and by Daude et al. [10] in the combined tight-binding and pseudopotential method. They give values of $0.6 \mathrm{eV}$ and $0.7 \mathrm{eV}$, respectively, which are about two times narrower than the full LMTO bandwidth and would probably be at odds with our experimental result after accounting for the experimental conditions. Our previous work [52], where we calculate the bandwidth using the linear combination of atomic orbital (LCAO) approximation in the framework of density-functional theory (DFT) with three different local density approximation Hamiltonians, predicts a range from 1.01 to $1.37 \mathrm{eV}$, a result, which is close to the present calculation. Unfortunately, there is no experimental data on the bandwidth of the $s$-band against which we can compare.

\subsection{2. $p$-Band}

We extracted the $p$-band dispersions in a similar manner to the $s$-band treatment and present the result in Fig. 8(a). The experimental data disperse from approximately 7.6 to $8.8 \mathrm{eV}$ whereas, the corresponding range for the LMTO data spreads from about 6.9 to $8.8 \mathrm{eV}$. It is evident from Fig. 8(a), that the two dispersion relationships are in fair agreement everywhere within the $0-1$ a.u. momentum interval, except for a small momentum range near the $\Gamma$ point. For momenta greater than 1 a.u. the experimental dispersion is essentially flat with only one shallow dip at about 1.7 a.u.. On the other hand, the LMTO result exhibits a wave- 
like pattern, with noticeable minima at about 1.7 a.u. and 2.5 a.u. for $q>1$ a.u.. However, there is good overall agreement of the experiment and theory throughout nearly the whole momentum range of 2.7 a.u..

The full LMTO bandwidth of the $p$-band is $2.97 \mathrm{eV}$, while the bandwidths extracted from the dispersion data in Fig. 8(a) are $1.87 \mathrm{eV}$ and $1.2 \pm 0.1 \mathrm{eV}$ for the LMTO and experiment, respectively. After additional simulation of the LMTO data to attempt to account for multiple scattering, the bandwidth becomes $1.22 \mathrm{eV}$. This value agrees with the experiment. To the best of our knowledge there are only two other experimental measurements for the width of the $p$-band. From the work of Doveren and Verhoeven [24] one can obtain a value of 3-4 eV. Fiermans et al. [23] observed a three-peak structure with an overall width of $9 \pm 0.5 \mathrm{eV}$. Both these values are substantially larger than our result.

The majority of available theoretical calculations predict a $p$-bandwidth value within 2-3 eV $[9,11,12,13,14,15,16,17,5,18,52]$. Our previous calculations [52] give a range from 2.61 to $2.88 \mathrm{eV}$, which is close to the present, raw LMTO result.

Normalized experimental and theoretical EMDs are presented in Fig. 8(b). Firstly, there is a striking discrepancy between the intensities at the $\Gamma$ point (about $60 \%$ and $4 \%$ of the maximum for the experiment and theory, respectively). The magnitude of this difference warrants that elastic scattering cannot be solely responsible. Secondly, the experimental EMD is at its maximum at around 0.6 a.u. whereas the theoretical maximum is about 0.8 a.u.. Thirdly, a high momentum tail is more intense by as much as a factor of 2 in the case of experiment compared to theory.

In Fig. 8(c) we present the density of occupied states (DOS) calculated by integration over the peak intensities. All three data sets are normalized to unity. The theory predicts that the DOS splits into two peaks separated by about $0.7 \mathrm{eV}$ (dashed curve). However, after the spectrometer resolution correction, the DOS is transformed into a single asymmetric peak 
(solid line) of a half-width of about $1.8 \mathrm{eV}$. The experimental DOS shows a similar asymmetric peak of a larger half-width $(2.6 \mathrm{eV})$ centred at $7.8 \mathrm{eV}$. The median energy of the $s$-band is $23.8 \mathrm{eV}$, and therefore, the measured $s-p$ band splitting is equal to $16 \mathrm{eV}$, which is in excellent agreement with the XPS data from the work of Fiermans et al. [23].

The experiment shows a significantly higher intensity in the $p$-band compared to the $s$-band than the LMTO calculation predicts. This is illustrated in Fig. 9 where the $p$-to-s band EMD ratio as a function of electron momentum is shown. In the momentum region from about 0.2 to 0.6 a.u., where the influence of multiple scattering on EMDs should be comparatively small, the experimental $p$-to-s band EMD ratio changes from 1.5 to 3 . The theory changes from 0.2 to 1.6 over the same momentum range. We observed a similar disagreement in the relative $p$ - to $-s$ - band $\mathrm{EMD}$ ratio for $\mathrm{MgO}[31]$ and $\mathrm{BeO}$ [51,52].

The experimental measurement and the LMTO calculation of the full valence bandwidths as the energy difference between the $s$ - and $p$-bands at the $\Gamma$ points $\left(\Gamma_{1} \rightarrow \Gamma_{15}\right)$ give values of $16.5 \pm 0.2 \mathrm{eV}$ and $14.41 \mathrm{eV}$, respectively. This calculated value also takes account of multiple scattering as described above. The bandwidth from the LMTO data spherically averaged and corrected with the experimental resolution is $15.43 \mathrm{eV}$. In this case, our attempt to account for multiple scattering leads to worse agreement between theory and experiment. Comparing our experimental value with the DFT-LCAO result from our previous work [52], we find that the full valence bandwidth of $16.7 \mathrm{eV}$ obtained in the B3PW scheme is the closest.

\section{2. $\quad$ Ca $3 p$ and $3 s$ Core Levels}

The Ca $3 p$ semi-core level shows much smaller dispersion than the $p$-band, but it is still noticeable due to the significant size of the Ca cations. We obtained the dispersion relation 
for this band in a similar manner to the oxygen valence bands and the result is shown in Fig. 10(a). The experimental band disperses from about $27.75 \mathrm{eV}$, at the $\Gamma$ point, to $28.4 \mathrm{eV}$, at 0.6 a.u.. The LMTO dispersion shows reasonable agreement with the experiment in the momentum interval from 0 to 0.9 a.u., with only a slight deviation from the experimental points near the $\Gamma$ point. Outside the $0-0.9$ a.u. momentum interval, there is only a qualitative resemblance of the dispersion relationship between the experiment and theory. However, it must be remembered that for these momenta there exists a significant smearing of the experimental intensity, due to elastic scattering, and the genuine dispersion relation is hard to establish.

Bandwidths, as determined from Fig. 10(a), are $0.7 \pm 0.1 \mathrm{eV}$ and $0.75 \mathrm{eV}$ for experiment and LMTO calculation, respectively. After applying an additional simulation of the LMTO data, to account for the effects of multiple scattering, the theoretical bandwidth decreases to $0.58 \mathrm{eV}$. With or without simulating multiple scattering the calculated bandwidths agree with experiment to within the error.

The magnitude of the experimental gap between the $s$-band and $\mathrm{Ca} 3 p$ semi-core level is $3.6 \pm 0.2 \mathrm{eV}$. This is slightly larger than the LMTO prediction of $3.15 \mathrm{eV}$, obtained after the effect of multiple scattering as well as the spherical averaging and experimental resolution was introduced into it. Raw LMTO gap is $2.76 \mathrm{eV}$, which suggests a decrease of $0.39 \mathrm{eV}$. Our previous gaps calculated using the DFT approach, with three different DFT Hamiltonians [52], range from 2.9 to $3.25 \mathrm{eV}$. Assuming a similar increase in the gap when multiple scattering is accounted for one obtains values ranging from 3.29 to $3.64 \mathrm{eV}$. The experimental value agrees well with this range.

The experimental and LMTO EMDs for the Ca $3 p$ semi-core level are shown in Fig. 10(b). Both data sets are normalized to unity at their respective maxima, which are at momenta of 0.75 a.u. and 0.65 a.u., for the experiment and theory, respectively. Due to 
multiple scattering and possibly other factors, the experimental EMD shows non-zero intensity (of the same magnitude as observed for the $p$-band) at the $\Gamma$ point.

The measured median energy for the $\mathrm{Ca} 3$ s core level is $46.9 \mathrm{eV}$, which results in a $\mathrm{Ca}$ $3 s-3 p$ core level splitting of $18.8 \pm 0.2 \mathrm{eV}$. The core level splitting measured by XPS $[22,24]$ yielded the value of $18.8 \mathrm{eV}$, in excellent agreement with our result.

The question arises as to whether there is any difference of the Ca $3 s-3 p$ splitting compared to metallic phase of calcium. We also measured the core levels for metallic $\mathrm{Ca}$ [33] and obtained a value of $19.4 \pm 0.5 \mathrm{eV}$. One can see that our splitting value, within the experimental error, is identical for the metallic and oxide phase of $\mathrm{Ca}$, which is also in agreement with the findings of Doveren and Verhoeven [24].

\section{Conclusions}

We have reported on the full energy - momentum resolved electronic structure of the valence band and the $\mathrm{Ca} 3 s$ and $3 p$ core levels in calcium oxide, measured using electron momentum spectroscopy. We have also compared our experimental results to the band structures calculated within the full potential linear muffin-tin orbital approximation, and to other available experimental and theoretical results.

Our experimental results for the dispersion relations in the $\mathrm{O}$ valence bands and $\mathrm{Ca} 3 p$ semi-core level show good general agreement with the LMTO calculations. The measured bandwidths for the oxygen $p$ - and $s$ - bands are $1.2 \pm 0.1 \mathrm{eV}$ and $0.6 \pm 0.2 \mathrm{eV}$, respectively. These values are quite different than the raw LMTO calculation which gives corresponding values of $2.97 \mathrm{eV}$ and $1.35 \mathrm{eV}$. Spherical averaging and correction of the LMTO calculation 
with the experimental resolution decrease the bandwidths but not enough to bring them into agreement with experiment. Our attempt to account for multiple scattering by simple additional convolution of the calculation improves the agreement. By contrast, the same approach gives worse agreement for the oxygen intervalence bandgap. The measured value is larger, by as much as $2.1 \mathrm{eV}$, than the LMTO prediction. However, our measured value for $s-p$ band splitting of $16 \mathrm{eV}$ agrees well with previous XPS data.

The EMD for the s-band determined from experiment is at odds with the theory prediction. The experimental EMD is higher throughout most of the $0-2$ a.u. momentum interval. It does not vanish at higher momenta as the result of multiple scattering. The $p$ band discrepancy is particularly apparent at the $\Gamma$ point, where the measured density is about $60 \%$ of the maximum, compared with near zero prediction of the theory. This value for our $\mathrm{BeO}$ and $\mathrm{MgO}$ experiment is about $30 \%$ of the maximum. The experiment also shows a considerably larger relative intensity of the $p$-band to the $s$-band, compared to the theory. Multiple scattering is considered to be responsible, partially, for the existing discrepancy in the $p$-band EMDs. However, the size of these differences are significant and could not be explained solely by the effect of multiple scattering.

There is good general agreement between the experiment and theory for the dispersion and EMD of the $3 p$ semi-core level. The measured bandwidth of $0.7 \pm 0.1 \mathrm{eV}$ does not contradict with the LMTO bandwidth, irrespective of whether multiple scattering is simulated in the calculated data. The measured $\mathrm{Ca} 3 s-3 p$ splitting of $18.8 \pm 0.2 \mathrm{eV}$ is in excellent accord with the existing XPS data.

Discrepancies in the intensity distributions, particularly the anomalous intensity in the $p$-bands at the $\Gamma$-point may be attributable, in part, to instrumental effects, as they have been observed in our previous measurements on $\mathrm{BeO}$ and $\mathrm{MgO}$. Although we have been careful to ensure that the spectrometer is properly aligned by measurements on carbon foils, we suspect 
that the measured intensity in the $p$-like bands is particularly sensitive to any such misalignment.

\section{Acknowledgments}

This work was supported by grants from the Australian Research Council and Flinders University of South Australia. One of us, MAB, would like to thanks the Shahid Bahonar University of Kerman for its support to be able to participate in this work. 


\section{FIGURE CAPTIONS}

Figure 1. Layout of (a) the scattering geometry of the (e,2e) spectrometer, and (b) target orientation with respect to incident and two outgoing electrons. The shaded sectors of cones in (a) indicate the angular acceptance of the analyzers. The shaded layer in (b) represents the portion of the target that contributes most to measured spectral momentum density.

Figure 2. A typical Auger spectrum (a) for a pure $\mathrm{Ca}$ surface and (b) of a $\mathrm{CaO}$ surface formed by calcium deposition in a $10^{-6}$ Torr oxygen atmosphere. Insets in both pictures display a magnifying view of the $\mathrm{Ca}_{2,3} \mathrm{VV}$ line energy region. The arrows indicate signals due to Auger electrons that experience a $35 \mathrm{eV}$ energy loss in $\mathrm{CaO}$.

Figure 3. Electron energy loss spectra for $\mathrm{CaO}$ targets prepared on 3-nm thick am-C substrates for (a) $19.6 \mathrm{keV}$ and (b) $1.2 \mathrm{keV}$ electrons. In (a) the solid line shows the fit result of the energy loss features.

Figure 4. Valence band and $\mathrm{Ca} 3 p$ semi-core level dispersions (two upper rows) and electron momentum densities (lower row) for $\mathrm{CaO}$ calculated within the FP-LMTO approximation along several high symmetry directions. The EMDs are plotted with the same line styles as used for the energy bands. The thick dashed line indicates the lower O $2 s$ band, the thin solid lines show the three upper $p$-bands and the dotted lines display the three lowest bands derived from the $\mathrm{Ca} 3 p^{6}$ semicore states. The first Brillouin zone is also shown.

Figure 5. Energy-momentum resolved densities in the valence band and Ca core levels of calcium oxide, (a) the spherically averaged and spectrometer resolution corrected LMTO 
calculations, (b) as measured by EMS, (c) obtained after subtraction of the am-C substrate contribution. The energy is referenced to the vacuum level of the spectrometer. The energy scale in (a) is shifted to match the minima of the $s$-bands in (a), (b) and (c). Density is depicted on a linear grey scale: darker shade corresponds to higher density.

Figure 6. Binding energy profiles for the valence band of calcium oxide. Results from the experiment (background subtracted) (points with error bars) and the spherically averaged and spectrometer resolution corrected LMTO calculation (solid lines) are shown. Intensities are normalized for the $s$-band at 0 a.u..

Figure 7. In (a), s-band dispersions, and in (b), EMDs, as extracted from the binding energy profiles of the experiment (points) and the spherically averaged and spectrometer resolution corrected LMTO calculation (solid line) for calcium oxide. In (b) both data sets are normalized to unity.

Figure 8. In (a), p-band dispersions, and in (b), EMDs, as extracted from the binding energy profiles from the experiment (points), and the spherically averaged and spectrometer resolution corrected LMTO calculation (solid line) for calcium oxide. In (c), the occupied DOS calculated from the momentum profiles of the experiment (points), and the spectrometer resolution corrected LMTO calculation (solid line) and the spherically averaged LMTO calculation (dash line). In (b) and (c) the experiment and LMTO data are normalized to unity. 
Figure 9. $P$ - to - $s$-band EMD ratio for calcium oxide extracted from the binding energy profiles of the experiment (points) and the spherically averaged and spectrometer resolution corrected LMTO calculation (solid line) as a function of electron momentum.

Figure 10. In (a), the Ca $3 p$ semi-core level dispersion, and in (b), EMDs, as extracted from the binding energy profiles of the experiment (points with error bars), and the spherically averaged and spectrometer resolution corrected LMTO (solid line) for calcium oxide. In (b) both densities are normalized to unity. 


\section{References}

\footnotetext{
${ }^{1}$ M.S.T. Bukowinski and R. Jeanloz, Geophys. Res. Lett. 7, 227 (1980).

${ }^{2}$ Handbook of Laser and Science and Technology, edited by M.J. Weber (CRC, Boca Raton, 1995), Supplement 2: Optical Materials.

${ }^{3}$ J.B. Torrance, Ph. Lacorre, C. Asavaroengchai, and R.M. Metzger, J. Solid State Chem. 90, 168 (1991); Phys.
} Stat. Sol. (c) 182, 351 (1991).

${ }^{4}$ I.V. Abarenkov and I.M. Antonova, Phys. Stat. Sol. B93, 315 (1979); J.B. Pendry, J. Phys. C10, 809 (1977).

${ }^{5}$ M.S.T. Bukowinski, J. Geophys. Res. B81, 303 (1982).

${ }^{6}$ G. Pacchioni, C. Sousa, F. Illas, F. Parmigiani, and P.S. Bagus, Phys. Rev. B48, 11573 (1993).

${ }^{7}$ F. Illas, A. Lorda, J. Rubio, J.B. Torrance, and P.S. Bagus, J. Chem. Phys. 99, 389 (1993).

${ }^{8}$ M. Posternak, A. Baldereschi, H. Krakauer, and R. Resta, Phys. Rev. B55, R15983 (1997); T. Kotani, Phys. Rev. B50, 14816 (1994); W.C. Mackrodt, N.M. Harrison, V.R. Saunders, N.L. Allan, M.D. Towler, E. Apra, and R. Dovesi, Phil. Mag. A 68, 653 (1993); L.F. Mattheiss, Phys. Rev. B5, 290 (1972).

${ }^{9}$ E.V. Stepanova, V.S. Stepanyuk, M.N. Rogaleva, O.V. Farberovich, A.A. Grigorenko, and V.V. Mikhalin, Sov. Phys. Solid State 30, 1329 (1988). Translated from Fiz. Tverd. Tela (Leningrad) 30, 2303 (1988).

${ }^{10}$ N. Daude, C. Jouanin, and C. Gout, Phys. Rev. B15, 2399 (1977).

${ }^{11}$ J. Redinger and K. Schwarz, Z. Physik B40, 269 (1981).

${ }^{12}$ U. Seth and R. Chaney, Phys. Rev. B12, 5923 (1975).

${ }^{13}$ M. Springborg and O.E. Taurian, J. Phys. C: Solid State Phys. 19, 6347 (1986).

${ }^{14}$ V.S. Stepanyuk, A. Szasz, A.A. Grigorenko, A.A. Katsnelson, O.V. Farberovich, V.V. Mikhalin, and A. Hendry, Phys. Stat. Sol. (b) 173, 633 (1992).

${ }^{15}$ R. Pandey, J.E. Jaffe, and A.B. Kunz, Phys. Rev. B43, 9228 (1991).

${ }^{16}$ G. Kalpana, B. Palanivel, and M. Rajagopalan, Phys. Rev. B52, 4 (1995).

${ }^{17}$ T. Kotani and H. Akai, Phys. Rev. B54, 16502 (1996).

${ }^{18}$ O.K. Andersen, H.L. Skriver, H. Nohl, and B. Johansson, Pure and Appl. Chem. 52, 93 (1979).

${ }^{19}$ M.J. Mehl, R.E. Cohen, and H. Krakauer, J. Geophys. Res. 93, 8009 (1988).

${ }^{20}$ C. Noguera, J. Goniakowski and S. Bouette-Russo, Surf. Sci. 287/288, 188 (1993).

${ }^{21}$ H. M. O’Bryan and H. W. B. Skinner, Proc. Roy. Soc. A176, 229 (1940).

${ }^{22}$ B. Demri and D. Muster, J. Mat. Proces. Techn. 55, 311 (1995). 
${ }^{23}$ L. Fiermans, R. Hoogewijs, G. de Meyer, and J. Vennik, Phys. Stat. Sol. (a) 59, 569 (1980).

${ }^{24}$ H. van Doveren and J.A.Th. Verhoeven, J. Elect. Spect. and Rel. Phen. 21, 265 (1980).

${ }^{25}$ R.C. Whited and W.C. Walker, Phys. Rev. 188, 1380 (1969).

${ }^{26}$ E. Garrone, A. Zecchina and F.S. Stone, Phil. Mag. B42, 683 (1980).

${ }^{27}$ E Weigold and I E McCarthy, Electron Momentum Spectroscopy, (New York: Kluwer Academic/Plenum Publishers, 1999)

${ }^{28}$ I.E. McCarthy and E. Weigold, Rep. Prog. Phys. 54, 789 (1991).

${ }^{29}$ M.A. Coplan, J.H. Moore, and J.P. Doering, Rev. Mod. Phys. 66, 985 (1994).

${ }^{30}$ J.R. Dennison and A.L. Ritter, J. Elect. Spect. and Rel. Phen. 77, 99 (1996); M. Vos and I.E. McCarthy, J. Elect. Spect. and Rel. Phen. 74, 15 (1995); Z. Fang, R.S. Matthews, S. Utteridge, M. Vos, S.A. Canney, X. Guo, I.E. McCarthy, and E. Weigold, Phys. Rev. B57, 12882 (1998); Z. Fang, X. Guo, S.A. Canney, S. Utteridge, M.J. Ford, I.E. McCarthy, A.S. Kheifets, M. Vos, and E. Weigold, Phys. Rev. B57, 4349 (1998); S.A. Canney, M. Vos, A.S. Kheifets, N. Clisby, I.E. McCarthy, and E. Weigold, J. Phys.: Condensed Matter 9, 1931 (1997); V.A. Sashin, S.A. Canney, M.J. Ford, M. Bolorizadeh, D.R. Oliver, and A.S. Kheifets, J. Phys.: Condens. Matter 12, 125 (2000).

${ }^{31}$ S.A. Canney, V.A. Sashin, M.J. Ford, and A.S. Kheifets, J. Phys.: Condens. Matter 11, 7507 (1999).

${ }^{32}$ X. Guo, S. Canney, A.S. Kheifets, M. Vos, Z. Fang, S. Utteridge, I.E. McCarthy, and E. Weigold, Phys. Rev. B54, 17943 (1996).

${ }^{33}$ V.A. Sashin, M.A. Bolorizadeh, A.S. Kheifets, and M.J. Ford, J. Phys.: Condens. Matter 12, 9407 (2000).

${ }^{34}$ S.A. Canney, M.J. Brunger, I.E. McCarthy, P.J. Storer, S. Utteridge, M. Vos, and E. Weigold, J. Electron Spectrosc. Relat. Phenom. 83, 65 (1997); P. Storer, R.S. Caprari, S.A.C. Clark, M. Vos, and E. Weigold, Rev. Sci. Instrum. 65, 2214 (1994).

${ }^{35}$ L.J. Allen, I.E. McCarthy, V.W. Maslen, and C.J. Rossouw, Aust. J. Phys. 43, 453 (1990).

${ }^{36}$ S.J. Utteridge, V.A. Sashin, S.A. Canney, M.J. Ford, Z. Fang, D.R. Oliver, M. Vos, and E. Weigold, App. Surf. Sci. 162/163, 357 (2000); Z. Fang, X. Guo, S. Utteridge, S.A. Canney and I.E. McCarthy, Rev. Sci. Instrum. 68, 4396 (1997).

${ }^{37}$ H. Gruner and G. Molenstedt, Thin Solid Films 1443 (1972).

${ }^{38}$ Practical Surface Analysis, volume 1, edited by D. Briggs and M.P. Seah (John Wiley \& Sons, Chichester, 1994). 
${ }^{39}$ P.Ascarelli and G. Missoni, J. Electron Spectros. Relat. Phenom., 5, 417 (1974); P.Ascarelli and G. Missoni, J. de Physique, Colloq. C2, 125 (1977).

40 J . Cazaux, J. Electron Spectros. Relat. Phenom. 105, 155 (1999).

${ }^{41}$ Handbook of Auger Electron Spectroscopy, edited by C.L. Hedberg (Physical Electronics, Inc., 1995).

${ }^{42}$ L.F. Mattheiss, Phys. Rev. B5, 306 (1972).

${ }^{43}$ E. Rudberg, Phys. Rev. 50, 138 (1936).

${ }^{44}$ E. Rudberg, Proc. Roy. Soc. A127, 111 (1930).

${ }^{45}$ A.R. Protheroe, A. Steinbrunn and T.E. Gallon, J. Phys. C: Solid State Phys. 15, 4951 (1982).

${ }^{46}$ A. S. Kheifets, D. R. Lun, and S. Y. Savrasov, J. Phys.: Condens. Matter 11, 6779 (1999).

${ }^{47}$ R. Wyckoff, Crystal Structures (New York: Interscience, 1963).

${ }^{48}$ M. Vos, P. Storer, A.S. Kheifets, I.E. McCarthy and E. Weigold, J. Electron Spectrosc. Relat. Phenom. 76103 (1995).

${ }^{49}$ B Soulé de Bas, H.E. Dorsett and M.J. Ford, J. Phys. Chem. Solids 64(3) 495 (2003)

${ }^{50}$ A.S. Kheifets, J. Lower, K.J. Nygaard, S. Utteridge, M. Vos, E. Weigold, and A.L. Ritter, Phys. Rev. B49, 2113 (1994).

${ }^{51}$ V.A. Sashin, M.A. Bolorizadeh, A.S. Kheifets and M.J. Ford, J. Phys.: Condens. Matter 15, 3567 (2003).

${ }^{52}$ V.A. Sashin, H.E. Dorsett, M.A. Bolorizadeh and M.J. Ford, J. Chem. Phys.113, 8175 (2000). 